\title{
An Investigation into the Collocations Used in the Translation of Official Documents from Persian into English
}

\author{
Mohammad Jafar Jabbari ${ }^{1, ~ *, ~ N a d i k a ~ K a v o o s i ~}{ }^{2}$ \\ ${ }^{1}$ Department of English, Yasouj University, Yasouj, Iran \\ ${ }^{2}$ English Department, School of Foreign Languages, Fars Science \& Research Branch, Islamic Azad University, Marvdasht, Iran
}

Email address:

mjjabbari@yu.ac.ir (M. J. Jabbari)

${ }^{*}$ Corresponding author

To cite this article:

Mohammad Jafar Jabbari, Nadika Kavoosi. An Investigation into the Collocations Used in the Translation of Official Documents from Persian into English. Communication and Linguistics Studies. Vol. 3, No. 2, 2017, pp. 15-21. doi: 10.11648/j.cls.20170302.11

Received: October 11, 2017; Accepted: November 17, 2017; Published: December 13, 2017

\begin{abstract}
A topic of paramount significance, yet underdeveloped in Translation Studies, is official translation. A fundamental problem that seems hazardous to the quality of official translation is deviant collocational structures produced by translators. Since official translationhas a highly significant stylistic and pragmatic part, any collocational errors can considerably affect the validity and readability of translations. The present researchaims tooffer a systematic study on the sources of collocationerrors made by certified translators. To do this, official texts and documents translated by the certified translators working at a number of translation bureaus of Shiraz, Iran, were investigated. The data collected were categorized according to Lie's model of error analysis, while the theoretical basis of the study was founded upon the assumptions of Functionalist linguistics. A statistical analysis of the data collected was also included. The findingsprovide some implications that can inspire future research both for the purpose of confirmation or exploration of newfindings.
\end{abstract}

Keywords: Collocation, Functionalism, Error Analysis, Translation Competence, Official Translation

\section{Introduction}

Renderingcollocations in the register of official translation can be especially challenging and perplexing in Translation Studies. A basic problem that may harm the quality of official translation is deviant collocational structures produced by translators. Since official translation plays a highly significant stylistic and pragmatic role, collocation errors can considerably affect the validity and readability of translations. The present study aims at presenting a systematic study on the sources of collocation errors made by certified translators. To this end, official texts and documents translated by the certified translators working at a number of translation bureaus of Shiraz, Iran, were collected and scrutinized carefully. The data collected were categorized according to [14] error analysis model. The theoretical basis of the study was founded upon the assumptions of Functionalist linguistics. A statistical analysis of the data collected was also included. The findings showed some implications that can inspire future research both for the purpose of assessment or exploration of new findings.

\section{Background}

Translation is one of the most dynamic language-based cultural encounters ([18]). Because cultures normally cut their perceptions of objects and concepts into specific and not usually universal lexical items, translating such items particularly culture-specific ones can bring challenging problems on the process of translation ([9]). This problem can reach even a more perplexing level when it comes to the construction and reproduction of idioms, fixed phrases, and collocations ([7]; [8]; [13]; [19]).

According to Functionalist theories of translation, each culture has constituted its own context-based language conventions, which are often explained within genres and registers ([9], [10]). Each register according to Halliday (as cited in [6], p. 38), "refers to consistent variations according to the 'use' of language."Now, if we consider the problem of collocations, according to such a Functionalist perspective, we learn that each register on its own might have developed a 
rich background of collocations and idiomatic conventions. Perhaps from among all of the common registers in languages, the "legal and official" register shows the highest tendency toward significance, recurrence, and fixity.

This especial significance of the legal discourse, however, can be simply ignored by those who are not well-aware of the textuality process in such a register. Translators dealing with official texts are among text-producers prone to making errors in the process of Official Translation (OT) ([11]). It should be noted, as some theorists such as Toury explain ([18]), translation from a particular stage becomes a text that belongs to the Target Language (TL) system. In other words, particular registers (especially non-fictional ones) are part of the pragmatic system of the TL. Considering this specific fact about translation, one may raise the question as to how the systemic patterns of official texts may be impaired if the collocational texture of such texts is ignored. This ignorance on the part of translators can lead to certain problems that are in fact the concerns of the present study. Therefore, the next section will provide a detailed account of the problems dealt with in this study.

\subsection{Definition of Key Terms}

\subsubsection{Collocation}

This language phenomenon can be described as the cooccurrence, cohesive relationship, and semantic construction of a combination of words that is normally recognized as fixed, recurrent, and idiomatic ([7]; [13]; [15]; [19]).

\subsubsection{Competence}

From a psychological perspective, language theorist contends that the process of language learning involves making new behaviors activated by a latent psychological construct. In TS, too, some theories hold the belief that the translator also experiences the development of psychological abilities, which are generally called competence [3]; [5].

\subsubsection{Error Analysis}

Error analysis is the systematic study of the nature and categories of errors normally committed by language learners. As learners go through psychological and developmental stages while learning the foreign language, they tend to make errors. Error analysis makes it possible to detect and classify these errors. [5]

\subsubsection{Official Translation}

Is the process of rendition of legal, notarized, and administrative documents or papers from one language into another. Nevertheless, as the sources show texts in such translations may thematically involve a wide range of topics that may go beyond a mere formal style or terminology ([5] and [7]).

\subsection{LinguisticCategorizations of Collocations}

Generally speaking, the formal mechanism of collocations from the point of view of linguistics can be divided into two distinct categories: grammatical and lexical collocations
([2]). The grammatical type of collocations normally involves "a dominant word (noun, adverb, and verb) and a preposition and a grammatical structure such as an infinitive or clause" ([2], p. 194). If put within formulaic expressions, the structures are as follows:

a) Noun + preposition

b) Noun + to + infinitive

c) Noun + that clause

d) Adjective + preposition

e) Predicate adjective + to + infinitive

f) Adjective + that clause

Nonetheless, the lexical collocations include specific formula, although they only include content words. The lexical violations in such structures will result in strange and even incomprehensive phrases. English speakers, for instance, may utter "close meetings" ([16] and O'Dell, 2005, p. 24), but a phrase such as "near meetings" may have a totally different or no meaning. The lexical structures for lexical collocations may include the following ([2], p. 197):

a) Verb + noun/pronoun

b) Verb + noun

c) Noun + verb

d) Adjective + noun

e) Noun + of + noun

f) Adverb + adjective

g) Verb + adverb

h) Noun + and + noun

\section{Purpose of the Study}

Considering the significance and perplexities of Official Translation (OT), the present researcher investigates the common types of errors committed by professional or official translators as far as collocations are concerned. To do this, the study follows a general framework developed to recognize the possible errors made by Second Language (L2) learners, and then framework will be adapted toerror detection in translating collocations (see [14]). The rationale behind the study is that there is a psychological mechanism that regulates the way translators or L2 leaners tend to reproduce collocations in the foreign language.

This mechanism can be best explained by [14] categorization of collocational errors, although only a certain number of the error types can be generalized to translating lexica items. Following that stage, the research will limit the investigation to collocational errors in official translations made by OT bureaus in Shiraz, Iran. In more specific words, the purposes of the research can be summarized as follows:

-To describe the most translation-related types of collocational error according to [14] categorization.

-To calculate the frequency of each of the categories of collocational errors and explain the implications that each category may have to clarify the functioning of collocational translation in official texts translated from Persian into English.

-To provide a glossary of the corrected collocations observed as errors, to list an illuminating sample of the cases 
in which the translated texts deviated from the collocational conventions of the TL.

\section{Data Collection}

The purpose of this study is to find possible regularities observed in the behavior of certified translators in rendering official collocations. To do this, the present researcher naturally relied on first-hand data collected from the translation bureaus in the city of Shiraz, Iran. More specifically, the data were collected through the random sampling method from the official documents translated by certified translators working at a number of the translation bureaus in Shiraz, Iran. To enhance the generalizability of the study, the researcher did not limit the translated texts to any thematically specific subject matter in OT. In other words, the data are an exact representative of all difficulties that certified translators experience in translating official collocations.

As a result, a varied collection of official collocations was gathered that may include legislative, punitive, administrative, procedural, and technical collocations, peculiar to official writing and composition. As it will be explained in the next part, this collection was based on originally Persian texts that were translated by certified the translators into English. Therefore, the pragmatic dimension of real-life use emphasized in Functionalism is obviously preserved in the data collected.

To enhance the validity of the translations, the researcher made sure that the texts were definitely translated by certified or in certain cases by professional translators employed at the translation offices under study. This would guarantee that the data were an exact representative of the work of professionally and socially responsible translators. It also highlights the need for studying collocation from a translational perspective, to trace any differences between professional language users and ordinary L2 leaners.

\section{Data Analysis}

In this section, all of the stages for carrying out the study will be reviewed in detail. After the data were collected, the researcher had to organize them systematically for intensive reading. After that, the collocations in the original texts were spotted and then compared to the choices made by the translators. Following that stage, the possible errors were categorized according to the model used in the study. The final findings were then presented as a glossary of corrected errors made by the translators.

\subsection{The Mechanism of Error Detection}

After intensive reading of the documents and their translations, the researcher faced three possibilities in categorizing the collocations found in the Persian text:

1. If the translator had replaced the Persian collocation with a correct one in English, the researcher would eliminate the collocation from the Persian collocation list, because no errors had been committed;

2. There were cases where the translator had totally ignored the collocation. This error is normally known as omission in TS ([17]). Because it was not primarily taken into account in [14] model of collocational EA, such cases were not considered in the final analysis. In reality, the purpose of the study is to discover the frequent errors made by professional translators in their actual translations. As a result, only translated items were taken into consideration in the analysis.

The cases which were actually translated as ill-formed collocations were seen as the basic data for analysis. In such cases, the researcher discovered that the translator had relied on a strategy to reproduce the collocation in question, but he or she had in reality failed to propose the correct collocation. In such cases the collocations were categorized according to the framework mentioned below. To determine collocational errors, the researcher relied on authentic legal sources translations such as ([4], [20] and [21]). To enhance the reliability of the findings, the data were validated by two experienced certified translators.

\subsection{The Categorization of the Errors Observed}

After the researcher was convinced that a translationrelated collocational error had been committed by the certified translated, she had to subsume the said error under a theoretically related source of error according to [14] basic categorization. However, because the model was primarily proposed for both lexical and grammatical errors some of the categories had to be eliminated for this study, which is focused only on lexical aspects of collocational errors. As a result, Overgeneralization and Ignorance of rule restrictions were not considered in the study, because they were proposed to discover grammatical sources of error.

Also, to simplify data analysis two of the lexicon-related categories were considered as one, for the reason that they shared fundamental similarities in detecting errors. These sources of error are Use of synonyms and Approximation. Because Approximation is the attempt to reproduce a collocation which "shares enough semantic features in common with the desire item to satisfy the speaker" ([20] as cited in [14], p. 491), it can at the same time cover the mechanism in Use of synonyms. In reality, even synonyms do not share exact semantic or pragmatic features. As a result, an attempt to reproduce any close semantic feature can at the same time be an instance of using synonyms.

Thus the elements, seen as sources of errors, in [14] basic framework are adapted to the purposes of the present study within this new framework.

\subsubsection{False Concepts Hypothesized}

Such errors result from learners' failure in comprehending distinctions in the TL. For example, a learner may imagine that such verbs as make and do have no specific meaning and they are as a result interchangeable. The leaner, having such a false idea, may say "make homework", instead of the normal collocation do homework. 
Interlingual transfer: As an infamous source of error, the learner's mother tongue can seriously affect his or her foreign language productions. As an instance, a Persian learner may produce "eat the pill", while the correct collocation in English is take the pill. Such false collocations can make a very strange image in the TL in such a way the audience may grasp something very differently from the intentions of the original writer.

\subsubsection{Word Coinage}

By making uneducated guesses, learners may try to create totally new vocabularies. As an instance, a Persian learner may say "sun-up" instead of sunrise. Clearly the source of error is not the Persian speaker's mother tongue, because such a collocation does not exist in Persian. It is in reality a total collocation creation.

\subsubsection{Approximation}

According to [20] (as cited in [14], p. 491), using an incorrect vocabulary item that "shares enough semantic features in common with the desire item to satisfy the speaker" is called approximation. For instance, the leaner, not familiar with the correct collocation, may produce "middle exam" instead of midterm.

As the final model for analysis, the sources of error in this study include false concepts hypothesized, Interlingual transfer, Word coinage, and Approximation.

TT. An analysis of the translated official texts in this study, for instance, showed that the certified translator had rendered the collocation /owraq-e tedzari/into "commercial papers". /?owraq-e tedzari/is itself a Persian collocation in which instead of other lexical possibilities /i.e. kaqæz, mædræk, siahe/ the term /? owraq/collocates with the adjective tejāri:

- /?owraq-e tedzari/

- papers-of-commercial

A certified translator normally encounters an official term such as papers in different register-related translations. In Persian the usual official equivalent for this term is /?owraq/. Apparently, the translator, influenced by prior technical knowledge of the English term papers, turned the Persian collocation into "commercial papers", while the correct TL collocation is commercial bills. As was explained in the literature review, such an error occurs when the translator assumes that there is no difference between the lexical items that in certain cases occupy comparable positions in collocation.

Another error found in the analyzed translated official texts was /tætbiq-e hesab-ha/, which was translated as "tally the accounts".

- /tætbiq-e hesab-ha /

- adjustment-of-accounts

/tætbiq/+ /hesab/is an official Persian collocation which denotes an activity that cannot be recognized by relying on an inductive analysis of individual lexical items. Clearly, the translator did not grasp the actual idea of the collocation by using the unrelated verb tally in the translation. The correct English collocation for this concept is reconciliation of accounts, which refers to tracing discrepancies in the accounts rather than matching the accounts. This translation results in a false hypo--research which can even complicate the translation and perplex the English speaking readers.

Another example is /suræt-e tæqiratdærvæz?-e mali/:

/suræt-e tæqirat dær væzS-e mali/

invoice-of-changes-at-station-of-financial

This phrase, which is a complex of two collocations, was translated as "invoice of changes in financial status". The Persian collocation refers to a technical term in official texts which demands an accurate English translation. "Invoice of changes and financial status, although both seemingly sound official phrases, are both deviant from the actual meaning intended by the original writer".

The translator used invoice instead of statement and status in place of position. As a result of this erroneouslycollocated translation, a falsely different concept may be inferred in English. The correct collocation is statement of changes in financial position. Clearly, such errors are a consequence of hasty decisions made according to TL prior knowledge. In the next category, however, a source of error will be analyzed that arises from the influence of the translator's mother tongue.

\subsubsection{Inter-lingual Transfer}

The native background knowledge that the translator carries with himself or herself is one of the most motivating facilitators of learning the foreign language. However, lack of linguistic or cultural knowledge in the TL can seriously damage translations if the translator is not conscious of crosslinguistic differences. The translator may reproduce concepts according to the way the SL cuts the world into lexical items. Since languages differ considerably in this aspect of lexicalization any ST transfer to the TT should be done with caution. The following instances show how easily professional translators may make inter-lingual errors.

/tænzim-e qærardad/is a normal Persian collocation:

/tænzim-e qærardad/

adjustment-of-contract

The Persian translator produced "organize the contract, which is a deviant collocation in the context. Clearly the translator was psychologically under the influence of Persian while concocting the English collocation. /tænzim/ in Persian refers to the process of making a contract between two parties. The translator, however, apparently used the first lexical item (organize) associated with /tænzim/ in his or her English mental vocabulary storage, creating a wrong collocation. The correct one is draw up the contract.

Another instance of interlingual transfer is the translation of /malekiat-e dowlæti/:

- /malekiat-e dowlæti/:

- owenership-of-governmental

This collocation connects /malekiat/ (instead of tæSælloq, Senhesar, or daraie) to /dowlæti/, creating a collocation which is more about society than government. Therefore, it hides a lexicalized need represented as a collocation. The TT, however, was totally based on a simple transfer of lexical items from SL into the TL, which resulted in "governmental 
ownership". The correct collocation is public ownership.

The translator simply activated the first lexical item associated with the word/dowlæti/, assuming that the concept is about governmental properties, while in reality it refers to public (people's) social facilities. This error which is mediated by the influence of Persian can disorient English readers, because they may not be able to find out why "government" is mentioned in an unrelated official context.

Another example which shows how linguistic prior expectations of translators can influence their TTs was found to be /shærik-e qeir-e fæSal/, meaning a nonexecutive provider of business funds:

/shærik-e qeir-e fæ?a/

partner-of-non-active

The translator in the texts under study had rendered this collocation into "inactive partner, which is totally unrelated collocation in English as a result of reliance on Persian semantic expectations. The correct collocation is silent partner, in which silent is used to mean "not taking part in an activity". In official translation silent is used in other collocations (i.e. silence of the law; silence gives consent), which shows that the word has special usages in collocational official terms.

This source of errors is a direct interference of translators' mother tongue lexical items and replacing them with the closest English item recognized by the translator. This type of error is still different from the next type which is an attempt to totally create new terms while they do not communicate much meaning to the audience. This other type is a consequence of the translator's ability in lexicalization and may not be related to her falsified concepts or mother tongue interference.

\subsubsection{Word Coinage}

Creating new lexical items is one of the basic capabilities of language users. Human beings are capable of coining new lexical items in case they need new ones. This creative process, however, can be exactly unproductive when a translator creates new vocabulary while there is no need for one. One of the causes of such an error is lack of command in register of the text being translated. In some cases the translator may produce lexical collocations that are totally new to the foreign audience, while there are genuine collocations in the TL to cover translation needs.

An example of coining new lexical items is /?ædæm-e ræ?aiat-emoqærrarat/, which is a strong collocation in Persian official texts:

/?ædæm-e ræSaiat-e moqærrarat/

lack-of-observance-of-regulations

The TT showed "non-observance of regulations, in which the translator made an attempt to invent a new lexical item according to the rules of English morphology. The correct collocation is failure to observe regulations.

Such an error is neither related to false concepts hypothesized nor to interlingual transfer, because firstly the translator was aware of the actual meaning of the ST collocation and secondly, he or she did not follow any Persian-related lexical constructions or semantics. Instead, the translator coined a new lexical item to cover the collocational structure seen in the Persian ST.

Another example is /pærdaxt be suræt-eperkeis/:

-/pærdaxt be suræt-e perkeis/

-payment-in-form-of-percase

In which /percejs/seems to be a false friend (see [19]) which has become a common term among Iranians; apparently, it was a result of a Persian combining of per and case (per+case) from the English formal structure. The translator of this collocation produced "pay as percase" which is an ill-correlated form collocation containing a coined item in the eyes' of English speaking readers. Asuggested well-correlated form, however, is simply per case.

Another instance of total word coinage was found in the translation of /gævahi-e Sefteqal be tæhsil/:

/gævahi-e ?efteqal be tæhsil/

certificate-of-employment-to-studying

This official term puts /e?fteqal/and/tæhsil/ together to form a register-based collocation, which was translated as "in-studying certificate". The logic of creating such a collocation is vaguely ambiguous, but it seems that in was used to mean during, in an attempt to reproduce the concept of /e? fteqal/. This translation, however, is hardly meaningful. The correct collocational phrase in English is student status certification.

As the examples analyzed indicate, word coinage appears to be an ultimate strategy in cases where the translator cannot find any equivalents in the TL, perhaps because of the culture-specific manner by which the collocation was framed in the SL. Although the already reviewed sources of error represent wide stylistic, semantic and collocational deviations, the next group concerns errors in which the translator did actually manage to recognize the semantic content, but mistakenly used a synonym for the correct collocational elements.

\subsubsection{Approximation}

As was explained above, words share certain semantic features. This lexical relation is known as synonymy. One of the basic problems with collocational constructions is that they cannot be substituted by their possibility synonymous equivalents. Due to this especial characteristic a lack of accuracy in reproducing collocations in translation may give an unusual sense to collocations. Translators who know a wide range of lexical items and synonyms are clearly prone to making errors when translating official texts, which are highly important as far as register and style, are concerned.

/hokm-e tælaq/, for instance, refers to a Persian legal collocation which is used to denote a non-lexicalized concept in the legal register:

/hokm-e tælaq/

verdict-of-divorce

It shows that instead of other Persian nouns (i.e. færman, dæstur, ?æmr), /hokm/ here collocates with /tælaq/. Also, this concept in reality refers to the objective document which indicates that divorce has been declared in a competent court of 
law. The Iranian certified translator had turned the collocation into "divorce verdict" in his (or her) English rendition. Because of the word verdict, which is an official term, one can suspect that the translator did have sufficient register knowledge in the TL, but he or she was not aware of the actual use of the collocation. The wrong strategy was to rely on a lexical item with similar semantic features: synonymy.

So the source of error is lack of knowledge of the registerrelated difference between verdict and bill in the English collocation expectation. The suggested well-correlated form in English is bill of divorcement, which is both semantically and stylistically different from the original collocation. Due to this wrong re-production of the Persian collocation, an approximate collocation is resulted that may complicate meaning in the TT.

Another collocation is /qanun-e ?æmri/, which conveys a particular status of legal condition:

/qanun-e ?æmri/

law-of-obligatory

The TT showed "obligatory law", which a deviant false concept in English. The translator, unaware of the difference between mandatory and obligatory as well as law and statute, attempted to give an approximation of the concept that may look meaningful superficially but totally unrelated in legal discourse. The suggested correct collocation here is mandatory statute.

Another Persian official collocation is /?ædæm-e ?esbate ?edde?a/:

/?ædæm-e ?esbat-e ?edde?a/

lack-of-proof-of-claim

Obviously, /?esbat/ and /?edde?a/ are strong Persian collocations, because they frequently occur with each other in the Persian legal discourse. This concept, too, refers to a special legal situation that cannot be inferred by the words individually. The TT was found to be "unproven claim, which is a total shift of the collocation (see [18], chap. 4). As was mentioned many times in the study, the collocations in OT are inherently different from those in other contexts. Hence, if they are re-written or wrongly conceptualized, they may convey deviant concepts.

In this case, too, although the shift shows the translator's knowledge of linguistic modifications, this strategy cannot help here because the collocation actually refers to a legal nonlexicalized status (the characteristic of collocations). The correct English collocation is failure of evidence, in which fail and evidence exhibit an unusual construction that cannot be simply predicted by the Persian translator. "Unproven claim", however, is a vaguely neighboring (approximate) phrase intended by the ST writer (for a discussion on this problem see 5.2. below).

The errors reviewed above all have something in common: they all show that the translators are competent and professional language users and they are not affected by their mother tongue. Their problem is obviously lack of registerbased collocational knowledge in English. It should be noted that translators possess a considerably rich reservoir of lexical knowledge. If this knowledgeis not validated according to register-based criteria, it can result in stylistically ill-formed translations (for a discussion on word style see [19], p. 88). On the other hand, one can claim that approximation may be to some limited extent different from other sources of error because it may not create widely deviant concepts (as opposed to false concepts hypothesized), and it is not the result of SL interference.

\section{Results}

In this section of the present chapter the results of the data analysis will be reported via quantitative statistical data. After the translated documents were analyzed for errors, the findings were categorized according to the four types of error as the theoretical framework of the study. Table 1 illustrates the frequency of each type.

Table 1. The Frequency of Each Type of Error as Observed in the Study.

\begin{tabular}{ll}
\hline Type of error & Frequency \\
\hline False concepts hypothesized & 34 \\
Interlingual transfer & 38 \\
Word coinage & 9 \\
Approximation & 61 \\
\hline
\end{tabular}

As Table 1 shows all of the sources of error in the study were discovered to have existed in the official translations made by the tested certified translators. The table also shows that 142 instances of errors were observed in the translations, while as can be seen the distribution of the data seems to be uneven across the sources of error.

Next, the data illustrated in the table can be standardized in terms of descriptive statistics to give a clearer image of the findings. After providing standardized data, the findings can be comparableagainstthose of other studies. Table 2 shows the standardized data.

Table 2. The Standardized Frequency of Each Type of Error as Observed in the Study.

\begin{tabular}{ll}
\hline Type of error & Frequency percentage \\
\hline False concepts hypothesized & 23.9 \\
Inter-lingual transfer & 26.8 \\
Word coinage & 6.3 \\
Approximation & 43.0 \\
\hline
\end{tabular}

The statistically standard data reveal the distribution of the errors in terms of percentage. Clearly, approximation (43.0\%) is the dominant source of error followed by interlingual transfer $(26.8 \%)$, false concepts hypothesized $(23.9 \%)$, and word coinage $(6.3 \%)$. Interestingly, approximation subsumes about half of the instances, although the other rough half is shared by the other sources of error. The important matter with the data is the possible reasons that might have resulted in such a data distribution. Of course this matter will be dealt with in detail in the next section of this chapter.

\section{Discussion}

The present research was concerned with studying the possible sources of error normally committed by certified translators in the process of translating Persian collocations 
into English. Due to the special nature of collocations L2 learners are prone to making various types of mistakes in reproducing collocations in the second language. The findings in L2 learning can considerably help translation researchers and trainers to take into account the possible sources of error complicating translators' work.

\section{Conclusion}

Deviant collocational structures produced by translators is a problem of paramount importance that may harm the quality of official translation. Owing to the importance of official translation, collocation errors can considerably affect the validity and readability of translations. The present study presented a systematic study on the sources of collocation errors made by certified translators. The data of the study were collected from the certified translation worked out by a number of translation bureaus within Shiraz, Iran, collected were classified according to [14] error analysis model. The theoretical basis of the study was founded upon the assumptions of Functionalist linguistics. A statistical analysis of the data collected showed that the most frequent errors were False concepts hypothesized, Inter-lingual transfer, Word coinage and Approximation, and their frequency percentage were, respectively $23.9,26.8,6.3$ and 43.0.

\section{Future Research}

This research works according to a systematic theoretical framework and theoretical basis. As a result, other studies can follow the procedures here to conduct new studies, both for the purpose of confirmation or further exploration. To provide some possibilities, some topics are mentioned in the following section for further research.

The findings of this study imply that the mechanism of committing errors in professional translators differ from those of L2 leaners. Researchers concerned with EA studies can compare the findings of this study to provide some insights into the validity of this assumption.

Also, the register-based nature of this study makes it possible to see translation as a contextualized competence. In other words, apparently each register may involve some hidden problems within itself. To discover what is actually happening, researchers can carry out other register-related studies and compare the findings tothose of this study. For instance, is the dominant problem of translating the medical register approximation or another source of error?

Another interesting topic for research is the relationship between shifts and collocations in translation. This study suggests that using shifts while translating collocations may deviate from standard language production. This seems to be a natural consequence, since collocations are fixed and semantically unpredictable constructions. So if the translator turns a noun into an adjective, the natural collocations construction may be damaged. As a result, a study could be devoted to the relationship between shifts and collocations in a specific register.

\section{References}

[1] Albir, A. H., \& Alves, F. (2009). Translation as a cognitive activity. In: J. Munday, (Ed.), Routledge companion to translation studies (pp. 54-73). New York, London: Routledge.

[2] Amiri, M. (2007). A course in vocabulary and reading comprehension $\left(5^{\text {th }}\right.$ Ed.). Tehran: Ketab-Arshad.

[3] Angelelli, C. V., \& Jacobson, H. E. (2009). Testing and assessment in translation and interpreting studies (Eds.). Amsterdam and Philadelphia: John Benjamins.

[4] Aslanzadeh, R. (1998). Practical Samples of the Translation of Documents and Records. Tehran, Samt.

[5] Bachman, L. F. (1990). Fundamental considerations in language testing. Oxford: Oxford University Press.

[6] Hatim, B. (2009). Translating text in context. In: J. Munday, (ed.), Routledge companion to translation studies (pp. 36-53). New York and London: Routledge.

[7] Hatim, B., and Mason, I. (1997). The translator as communicator. London and New York: Routledge.

[8] Hatim, B., and Munday, J. (2004). Translation: An advanced resource book. New York and London: Routledge.

[9] House, J. (2001). Translation quality assessment: Linguistic description versus social evaluation. Meta, 46, 2, 243-256.

[10] House, J. (2009). Translation. Oxford: Oxford University Press.

[11] Jabbari, M. J. (1991). A surface register contrastive analysis of officially translated documents into English. Unpublished master's thesis, Shiraz University, Shiraz, Iran.

[12] Keshavarz, M. H. (1999). Contrastive analysis and error analysis. Tehran: Rahnama Publication.

[13] Larson, M. (1998). Meaning-based translation: A guide to cross-language equivalence $\left(2^{\text {nd }} \mathrm{ed}.\right)$. Lanham: University Press of America.

[14] Liu, C. P. (1999). An analysis of collocation errors in EFL writing. The Proceedings of the English International Symposium on English Teaching (pp. 483-494). Taipei: Crane Publishing Ltd.

[15] Martynska, M. (2004). Do English learners know collocations? Investigationeslinguisticae, 60, pp. 2-12.

[16] McCarthy, M., \& O’Dell, F. (2005). English collocations in use. Cambridge: Cambridge University Press.

[17] Melis, N. M., \& Albir, A. H. (2001). Assessment in translation studies: Research needs. Meta, 46, 2, 272-287.

[18] Munday, J. (2012). Introducing translation studies: Theories and application ( $3^{\text {rd }}$ ed.). New York and London: Routledge.

[19] Newmark, P. (1988). A textbook of translation. New York and London: Prentice Hall.

[20] Tavangar, H. (1998). Translation of Documents and Records. $1^{\text {st }}$ Vol. Tehran, Payam-e-Noor Press.

[21] Tavangar, H. (1999). Translation of Documents and Records. $2^{\text {nd }}$ Vol. Tehran, Payam-e-Noor University Press. 